\title{
Non-invasive computed tomography coronary angiography as a gatekeeper for invasive coronary angiography
}

\author{
Fleur R. de Graaf · Joëlla E. van Velzen · Stephanie M. de Boer • \\ Jacob M. van Werkhoven - Lucia J. Kroft • Albert de Roos • Allard Sieders • \\ Greetje J. de Grooth · J. Wouter Jukema · Joanne D. Schuijf · Jeroen J. Bax • \\ Martin J. Schalij $\cdot$ Ernst E. van der Wall
}

Received: 3 July 2011 / Accepted: 20 April 2012/Published online: 11 May 2012

(C) The Author(s) 2012. This article is published with open access at Springerlink.com

\begin{abstract}
To determine the rate of subsequent invasive coronary angiography (ICA) and revascularization in relation to computed tomography coronary angiography (CTA) results. In addition, independent determinants of subsequent ICA and revascularization were evaluated. CTA studies were performed using a 64-row $(n=413)$ or 320-row $(n=224)$ multidetector scanner. The presence and severity of CAD were determined on CTA. Following CTA, patients were followed up for 1 year for the occurrence of ICA and revascularization. A total of 637 patients (296 male, $56 \pm 12$ years) were enrolled and 578 CTA investigations were available for analysis. In patients with significant CAD on CTA, subsequent ICA rate was $76 \%$. Among patients with non-significant CAD on CTA, subsequent ICA rate was $20 \%$ and among patients with normal CTA results, subsequent ICA rate was $5.7 \%$ $(p<0.001)$. Of patients with significant CAD on CTA,
\end{abstract}

F. R. de Graaf $(\bowtie)$. J. E. van Velzen .

S. M. de Boer · J. M. van Werkhoven · G. J. de Grooth .

J. W. Jukema · J. D. Schuijf · J. J. Bax .

M. J. Schalij · E. E. van der Wall

Department of Cardiology, Leiden University Medical Center, Albinusdreef 2, 2333 ZA Leiden, The Netherlands

e-mail: f.r.de_graaf@lumc.nl

J. E. van Velzen · J. M. van Werkhoven .

J. W. Jukema - E. E. van der Wall

The Interuniversity Cardiology Institute of the Netherlands,

Utrecht, The Netherlands

\section{J. Kroft · A. de Roos}

Department of Radiology, Leiden University Medical Center, Leiden, The Netherlands

A. Sieders

Department of Cardiology, Rijnland Hospital, Leiderdorp,

The Netherlands revascularization rate was $47 \%$, as compared to a revascularization rate of $0.6 \%$ in patients with non-significant CAD on CTA and no revascularizations in patients with a normal CTA results $(p<0.001)$. Significant CAD on CTA and significant three-vessel or left main disease on CTA were identified as the strongest independent predictors of ICA and revascularization. CTA results are strong and independent determinants of subsequent ICA and revascularization. Consequently, CTA has the potential to serve as a gatekeeper for ICA to identify patients who are most likely to benefit from revascularization and exclude patients who can safely avoid ICA.

Keywords Cardiac imaging - Coronary artery disease . Multidetector computed tomography · Invasive coronary angiography

\section{Introduction}

Invasive coronary angiography (ICA) is routinely used for the identification of patients with suspected coronary artery disease (CAD). Advantages of ICA are high resolution imaging and the possibility of revascularization by percutaneous coronary intervention (PCI). Due to its invasive nature, ICA is associated with a small risk of complications, radiation exposure and relatively high cost of hospital stay. Additionally, the rate of normal ICA examinations is still quite high and health-care costs associated with the increase in ICA and revascularization rates are substantial. Moreover, a recent multicenter study showed that PCI has no superiority over pharmacological therapy in patients with stable CAD [1]. Accordingly a non-invasive test to select the most suitable patients for ICA and revascularization would be preferable. Most 
traditional non-invasive cardiac imaging techniques rely on the detection of stress-inducible ischemia [2]. However, with the introduction of computed tomography coronary angiography (CTA), the non-invasive anatomic assessment of CAD with high diagnostic accuracy has become possible. Prior studies have shown that CTA allows reliable patient risk stratification, and normal CTA examinations indicate good prognosis [3, 4]. Although CTA cannot replace ICA, this technique could serve as a gatekeeper for ICA in selected patients, and thus avoid unnecessary additional examinations. At the same time concerns have been raised that CTA may trigger unnecessary referral for ICA. Rates of ICA and interventional therapy following CTA have been largely unreported. The purpose of the present study therefore was to determine the rate of subsequent ICA and revascularization in relation to CTA results. Furthermore, independent determinants of subsequent ICA and revascularization were investigated.

\section{Methods}

\section{Patient population}

The study group consisted of patients who were referred for CTA as part of a large ongoing registry exploring the prognostic value of CTA [5]. Reasons for referral were typical chest pain, atypical chest pain and non-anginal chest pain, according to the appropriate use criteria for cardiac computed tomography [6]. Exclusion criteria for CTA investigation were: renal insufficiency (glomerular filtration rate $<30 \mathrm{ml} / \mathrm{min}$ ), (supra)ventricular arrhythmias, known allergy to iodine contrast material, severe claustrophobia, pregnancy and high heart rate in the presence of contraindications to $\beta$-blocker medication [7]. Patients were entered prospectively into the departmental patient information system (EPD-Vision ${ }^{\circledR}$, Leiden University Medical Center) and retrospectively analysed. Patients with known CAD or congenital cardiac abnormalities were excluded from the study.

\section{CTA data acquisition}

CTA studies were performed using a 64-row $(n=413)$ or 320-row ( $\mathrm{n}=224$ ) multidetector scanner (Aquilion 64, and Aquilion ONE, Toshiba Medical Systems, Otawara, Japan) with 64 and 320 simultaneous detector rows, respectively (each $0.5 \mathrm{~mm}$ wide), as previously described [8, 9]. One hour before the investigation, oral $\beta$-blocker medication (metoprolol 50 or $100 \mathrm{mg}$ ) was administered to patients with a heart rate $\geq 65$ beats/min, unless contra-indicated. The total amount of non-ionic contrast media (Iomeron 400; Bracco, Milan, Italy) injected into the antecubital vein was
60-100 $\mathrm{ml}$ (depending on scanner type and body weight) at a flow rate of $5.0-6.0 \mathrm{ml} / \mathrm{s}$. In order to synchronize the arrival of the contrast media, bolus arrival was detected using a real-time bolus tracking technique. All images were acquired during a single inspiratory breath-hold of maximally $12 \mathrm{~s}$ for 64 row-CTA and $5 \mathrm{~s}$ for 320-row CTA. For 64-row CTA, a helical-scanning technique was used as previously described [10]. In brief, during the examination the ECG was registered simultaneously for retrospective gating of the data. A collimation of $64 \times 0.5 \mathrm{~mm}$ was used. During 320-row CTA, the ECG was registered simultaneously for prospective triggering of the data. A collimation of $320 \times 0.5 \mathrm{~mm}$ was used and the entire heart was imaged in a single heart beat, as previously reported [11].

The estimated mean radiation dose for 64-row CTA was $18.1 \pm 5.9 \mathrm{mSv}$ in patients scanned using retrospective ECG gating. The estimated mean radiation dose for 320-row CTA was $3.2 \pm 1.1 \mathrm{mSv}$ if scanned ful-dose at $75 \%$ of the cardiac cycle. In patients who were scanned full-dose at $65-85 \%$ of the R-R interval, estimated mean radiation dose was $7.1 \pm 1.7 \mathrm{mSv}$.

\section{CTA data analysis}

Data were transferred to a remote workstation with dedicated analysis software (for 64-row CTA reconstructions: Vitrea 2; for 320-row CTA reconstructions: Vitrea FX 2.0, Vital Images, Minnetonka, MN, USA). First, calcium score was assessed and an overall Agatston score was registered for each patient. Next, coronary arteries were evaluated as previously described [8]. Presence of CAD was assessed as recommended by the SCCT guidelines for the interpretation and reporting of CTA [12]. Each scan classified as having (1) normal, (2) non-significant CAD (luminal narrowing $<50 \%$ in diameter), (3) obstructive CAD ( $\geq 50 \%$ luminal narrowing), as described [13]. In addition, the presence of significant left main disease and significant three-vessel disease was noted. After data evaluation, CTA results were entered in into the departmental Cardiology Information System (EPD-Vision ${ }^{\circledR}$ ) without recommendations for further clinical management. Further clinical management was determined at the discretion of the referring cardiologist.

\section{ICA and revascularization}

ICA was performed according to standard techniques. Following CTA, patients were followed up for 1 year for the occurrence of ICA and revascularization. Patient follow-up information was obtained by one observer, blinded to the baseline CTA results, using data from clinical visits and/or standardized telephone interviews. 
Statistical analysis

Statistical analysis was performed using SPSS software (version 16.0, Inc., Chicago, Illinois). Quantitative data were expressed as mean \pm standard deviation (SD). Categorical variables were described as numbers and percentages and comparison was performed by Chi-square test. Univariate analysis of clinical baseline variables and significant CAD on CTA was performed. For each variable, odds ratio (OR) and $95 \%$-confidence interval (CI) were calculated. Subsequently, multivariate logistic regression analysis for ICA and revascularization were performed (using backward elimination method with $p$-value $>0.2$ as the criterion for elimination) to determine the independent association with significant CAD on CTA and significant three-vessel or left main disease on CTA, each corrected for clinical baseline variables (age, gender, diabetes, hypercholesterolemia, hypertension, family, smoking and obesity) in a separate model. A $p$ value $<0.05$ was considered statistically significant.

\section{Results}

Study population

A total of 637 patients were enrolled in the study population. An overview of the patient characteristics is shown in Table 1 . In brief, $47 \%$ of patients were male with a mean

Table 1 Clinical characteristics $(n=637)$

\begin{tabular}{ll}
\hline Age (years) & $56 \pm 12$ \\
Men/women & $296 / 341$ \\
Diabetes & $19 \%$ \\
Hypercholesterolemia $^{\mathrm{a}}$ & $34 \%$ \\
Hypertension $^{\mathrm{b}}$ & $43 \%$ \\
Family history of CAD $^{\mathrm{c}}$ & $46 \%$ \\
Smoking $^{\text {Obesity }}{ }^{\mathrm{d}}$ & $20 \%$ \\
Reason of referral for CTA $^{\text {Typical chest pain }}$ & $21 \%$ \\
Atypical chest pain & \\
Non-anginal chest pain & $21 \%$ \\
\hline
\end{tabular}

Data are absolute values, percentages or means \pm standard deviation $B M I$ body mass index, $C A D$ coronary artery disease, CTA computed tomography coronary angiography

a Serum total cholesterol $\geq 230 \mathrm{mg} / \mathrm{dl}$ and/or serum triglycerides $\geq 200 \mathrm{mg} / \mathrm{dl}$ or treatment with lipid lowering drugs, ${ }^{\mathrm{b}}$ Defined as systolic blood pressure $\geq 140 \mathrm{~mm} \mathrm{Hg}$ and/or diastolic blood pressure $\geq 90 \mathrm{~mm} \mathrm{Hg}$ and/or the use of antihypertensive medication, ${ }^{\mathrm{c}}$ Defined as presence of coronary artery disease in first degree family members at $<55$ years in men and $<65$ years in women, ${ }^{d}$ Defined as a BMI $\geq 30 \mathrm{~kg} / \mathrm{m}^{2}$ age of $56 \pm 12$ years. Reasons for referral were typical chest pain in $21 \%$, atypical chest pain in $46 \%$ and nonanginal chest pain in $33 \%$.

A total of 27 scans $(4.2 \%)$ were of non-diagnostic image quality, and excluded from the analysis. The presence of blooming artifacts in patients with a high calcium score $\geq 400$ accounted for 7 uninterpretable scans. Furthermore, 30 patients $(3.8 \%)$ were lost to follow-up and 2 patients died before follow up was completed. As a result, a total of 578 patients were included in the analysis.

\section{CTA results}

In a total of 578 patients, CTA results were normal in 212 patients $(37 \%)$, non-significant CAD was observed in 177 patients $(30 \%)$ and significant $(\geq 50 \%)$ CAD was identified in 189 patients (33\%). Additionally, significant threevessel or left main disease on CTA was observed in 34 patients $(5.9 \%)$, while the presence of significant threevessel or left main disease could not be determined in two patients due to insufficient image quality.

ICA

Subsequent to CTA, ICA was performed in 190 patients (33\%). The mean duration between CTA and ICA was $2.6 \pm 2.7$ months. Of the 189 CTA investigations with significant CAD, subsequent ICA rate was $76 \%(n=143)$. Among 177 patients with non-significant CAD on CTA, subsequent ICA rate was $20 \%(\mathrm{n}=35)$ and among 212 patients with normal CTA results, subsequent ICA rate was $5.7 \%(\mathrm{n}=12 ; p<0.001)$. Figure 1 illustrates the relationship between CTA results and subsequent ICA. Moreover, of the 34 patients with significant three-vessel or left main disease on CTA, subsequent ICA rate was $88 \%(n=30)$, while ICA rate in 542 patients without significant three-vessel or left main disease on CTA was $29 \%(\mathrm{n}=158, p<0.001)$.

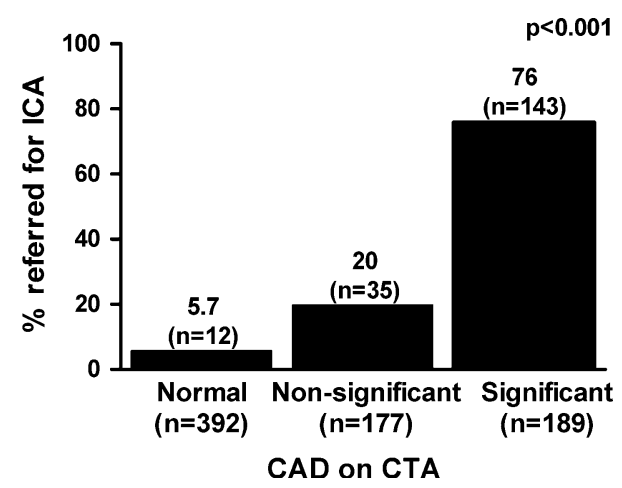

Fig. 1 Bar graph illustrating the relationship between degree of CAD on CTA and subsequent referral for ICA. CAD coronary artery disease, CTA computed tomography coronary angiography, ICA invasive coronary angiography 
Univariate regression analysis was performed to identify determinants of subsequent ICA. Table 2 shows that significant CAD on CTA (OR 22.62) as well as significant three-vessel or left main disease on CTA (OR 18.23) were identified as a significant univariate determinant of subsequent ICA. Furthermore, the clinical baseline variables age, gender, hypercholesterolemia, hypertension and smoking were significant univariate determinants of ICA.

Subsequently, multivariate logistic regression analysis for ICA was performed to determine the independent association with significant CAD on CTA and significant three-vessel or left main disease on CTA, each corrected for clinical baseline variables in a separate model. Significant CAD on CTA (OR 18.60) and significant three-vessel or left main disease on CTA (OR 15.67) were identified as the strongest independent predictors of ICA. Other determinants of ICA of lesser statistical significance were gender and smoking. Table 2 shows the results of uni- and multivariate regression analysis to identify determinants of subsequent ICA.
Revascularization

A total of 89 patients (15\%) underwent revascularization, of whom 74 patients underwent PCI and 15 patients coronary artery bypass grafting (CABG). Of the 189 patients with significant $\mathrm{CAD}$ on $\mathrm{CTA}$, revascularization rate was $47 \%(\mathrm{n}=88)$, as compared to a revascularization rate of $0.6 \%(\mathrm{n}=1)$ in 348 patients with non-significant CAD on CTA. Of note, this patient had a significant lesion in the distal RCA, which was underestimated on CTA. No revascularizations were performed in patients with a normal CTA examination $(p<0.001)$. The frequency of revascularization in relation to CAD on CTA is illustrated in Fig. 2.

In 34 patients with significant three-vessel or left main disease on CTA, revascularization rate was $68 \%(n=23)$, as compared to $12 \%(\mathrm{n}=64)$ in 542 patients without significant three-vessel or left main disease on CTA $(p<0.001)$. Table 2 shows that significant CAD on CTA (OR 338.06) as well as significant three-vessel or left main

Table 2 Independent determinants of subsequent ICA and revascularization

\begin{tabular}{|c|c|c|c|c|}
\hline \multirow[t]{2}{*}{ Variable } & \multicolumn{2}{|l|}{ Univariate } & \multicolumn{2}{|l|}{ Multivariate } \\
\hline & OR $(95 \% \mathrm{CI})$ & $p$-value & OR $(95 \% \mathrm{CI})$ & $p$-value \\
\hline \multicolumn{5}{|l|}{ ICA } \\
\hline Age & $1.05(1.03-1.06)$ & $<0.001$ & $1.02(0.99-1.04)$ & 0.112 \\
\hline Gender & $1.92(1.35-2.73)$ & $<0.001$ & $1.81(1.13-2.91)$ & 0.014 \\
\hline Diabetes & $1.35(0.87-2.08)$ & 0.182 & - & - \\
\hline Hypercholesterolemia & $2.19(1.53-3.14)$ & $<0.001$ & $1.42(0.87-2.30)$ & 0.162 \\
\hline Hypertension & $2.09(1.47-2.98)$ & $<0.001$ & $1.51(0.93-2.46)$ & 0.098 \\
\hline Family history of CAD & $0.83(0.58-1.17)$ & 0.282 & - & - \\
\hline Smoking & $2.70(1.78-4.09)$ & $<0.001$ & $2.35(1.33-4.14)$ & 0.003 \\
\hline Obesity & $1.08(0.69-1.67)$ & 0.749 & - & - \\
\hline Significant CAD on $\mathrm{CTA}^{\mathrm{a}}$ & $22.62(14.41-35.51)$ & $<0.001$ & $18.60(11.46-30.19)$ & $<0.001$ \\
\hline Significant three-vessel or left main disease on $\mathrm{CTA}^{\mathrm{a}}$ & $18.23(6.32-52.59)$ & $<0.001$ & $15.67(4.59-53.43)$ & $<0.001$ \\
\hline \multicolumn{5}{|l|}{ Revascularization } \\
\hline Age & $1.05(1.03-1.07)$ & $<0.001$ & $1.02(0.99-1.06)$ & 0.134 \\
\hline Gender & $2.80(1.73-4.53)$ & $<0.001$ & $2.90(1.54-5.46)$ & 0.001 \\
\hline Diabetes & $2.08(1.24-3.49)$ & 0.005 & $2.10(1.00-4.43)$ & 0.050 \\
\hline Hypercholesterolemia & $2.31(1.46-3.66)$ & $<0.001$ & $1.45(0.78-2.69)$ & 0.243 \\
\hline Hypertension & $1.92(1.22-3.04)$ & 0.005 & - & - \\
\hline Family history of CAD & $0.67(0.42-1.07)$ & 0.095 & - & - \\
\hline Smoking & $3.43(2.11-5.58)$ & $<0.001$ & $3.24(1.60-6.57)$ & 0.001 \\
\hline Obesity & $1.09(0.62-1.92)$ & 0.773 & - & - \\
\hline Significant CAD on $\mathrm{CTA}^{\mathrm{a}}$ & $338.06(46.53-2,456.30)$ & $<0.001$ & $282.61(38.21-2,090.31)$ & $<0.001$ \\
\hline Significant three-vessel or left main disease on $\mathrm{CTA}^{\mathrm{a}}$ & $15.62(7.27-33.54)$ & $<0.001$ & $12.31(5.52-28.91)$ & $<0.001$ \\
\hline
\end{tabular}

$C A D$ coronary artery disease, $C T A$ computed tomography coronary angiography, ICA invasive coronary angiography

${ }^{a}$ Each variable was included in a separate model corrected for clinical baseline variables (age, gender, diabetes, hypercholesterolemia, hypertension, family, smoking and obesity). Results from multivariate analysis for clinical baseline variables shown in the table were derived from the model including significant CAD on CTA 


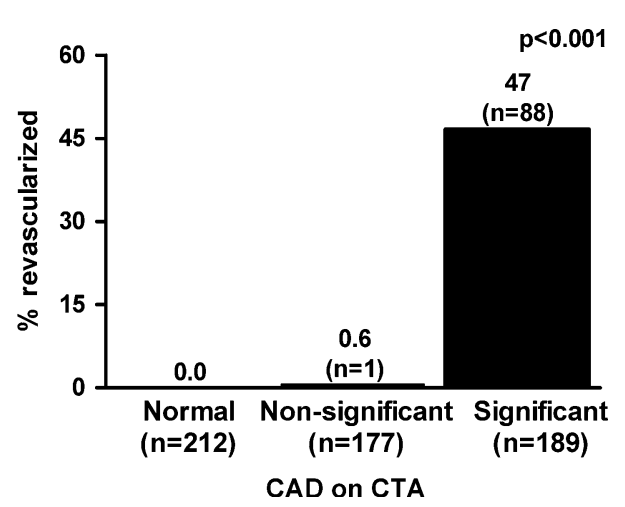

Fig. 2 Bar graph illustrating the relationship between degree of CAD on CTA and revascularization. CAD coronary artery disease, CTA computed tomography coronary angiography

disease on CTA (OR 15.62) were identified as significant determinants of revascularization in univariate analysis. Furthermore, the clinical baseline variables age, gender, hypercholesterolemia, hypertension and smoking were significant univariate determinants of revascularization.

Next, multivariate logistic regression analysis for revascularization was performed to determine the independent association of significant CAD on CTA and significant three-vessel or left main disease on CTA, each corrected for clinical baseline variables in a separate model. Multivariate regression analysis identified significant CAD on CTA (OR 282.61) and significant threevessel or left main disease on CTA (OR 12.31) as the strongest predictors of revascularization. Additional significant determinants were gender and smoking. In Table 2, the results of uni- and multivariate regression analysis to identify determinants of revascularization are shown.

\section{Discussion}

The present clinical investigation evaluated the association between CTA results and subsequent rates of ICA and revascularization. The majority of patients with significant CAD on CTA were referred for subsequent ICA (76\%), while in patients with normal CTA results a very low rate of referral was demonstrated $(5.7 \%)$. Additionally, no patients with normal CTA results underwent revascularization. Moreover, significant CAD and significant threevessel or left main disease on CTA were identified as the strongest independent determinants of subsequent ICA and revascularization.

\section{Previous literature}

The use of CTA to reliably exclude significant CAD is supported by extensive literature validating this technique against ICA [14]. Nevertheless, limited information is available regarding the influence of CTA results on clinical decision making and referral for downstream testing such as ICA. Henneman and colleagues previously showed that a substantial proportion of patients with suspected CAD have normal coronaries on CTA examination [15]. As a result, in a substantial percentage of patients with suspected CAD, significant stenosis may be excluded using CTA. Furthermore, Chow et al. [16] recently studied the clinical impact of CTA on the rate of normal ICA. In a large cohort of 7,017 consecutive patients who were referred for ICA before and after implementation of a dedicated CTA program, the implementation of CTA had a positive effect on ICA referral by reducing the frequency of normal ICA from 32 to $27 \%$. The present results expand on these findings, in identifying a strong association between CTA results and referral for ICA. Moreover, the current findings showed a high percentage of normal and non-significant CT results. Considering that normal CTA examinations are associated with a good prognosis [17], these data imply that, using CTA, a large proportion of patients with chest pain or a high risk profile may be safely excluded from ICA.

Even though significant CAD on CTA was the strongest predictor for revascularization, still a considerable proportion of patients $(24 \%)$ with significant CTA results were not referred for ICA. Similarly, a small percentage of patients with non-significant and normal CTA results (20 and $5.7 \%$, respectively) were referred for ICA. These findings could be explained by the fact that other clinical information and test results, such as exercise ECG or myocardial perfusion imaging (MPI), may have also influenced referral for ICA. Indeed, clinical presentation and functional information also influence subsequent referral to ICA and revascularization. While no previous studies have investigated ICA rates in relation to CTA results, a prior investigation by Bateman and colleagues showed comparable ICA referral rates in patients who were referred for MPI using single photon emission computed tomography (SPECT) [18]. In a group of 4,162 patients with a mean follow up of 8.9 months, $60 \%$ of patients with high-risk ischemia were referred for ICA, as compared with $9 \%$ with mild ischemia and $3.5 \%$ of patients without ischemia on SPECT. In this population, $40 \%$ of high-risk patients were not referred for invasive imaging, most likely due to the fact that other clinical information and previous study results also influenced patient management. A more recent study by Shaw et al. [19] showed comparable results. In analyzing post-SPECT referral rates, $52 \%$ of patients with 3 ischemic perfusion areas underwent ICA. Unfortunately, studies directly comparing CTA and MPI are not available, and future investigations are warranted. 
Anatomical and functional imaging prior to ICA

Most traditional non-invasive cardiac imaging techniques rely on the detection of stress-inducible ischemia $[18,20$, 21]. In this setting, perfusion abnormalities or systolic dysfunction serve as surrogate markers for flow-limiting CAD [22]. Although CTA and MPI (the most frequently applied functional imaging technique) provide complementary information [22], concerns about radiation exposure preclude the use of both CTA and MPI in all patients. With the introduction of CTA, the use of MPI as a gatekeeper for ICA has been challenged [23]. First, CTA has a negative predictive value approaching $100 \%$, making it an excellent modality for the exclusion of CAD in patients with a low-to-intermediate pre-test likelihood. Conversely, MPI enables the identification of perfusion abnormalities, due to which this modality is particularly suitable for ruling in $\mathrm{CAD}$, especially in higher risk patients or patients with unknown CAD [24]. Thus, individual patient characteristics are important in the choice of non-invasive imaging modality to further guide patient management. Second, while both MPI and CTA are associated with radiation exposure, radiation exposure of CTA has been substantially reduced using novel low-dose algorithms. In daily clinical practice, however, the choice of non-invasive imaging modality prior to ICA may also depend on availability [20] and local expertise. Finally, with the large increase in health-care costs focus is increasingly shifting to costeffective use of resources. Preliminary results suggest that costs of CTA as a gatekeeper for ICA may be significantly lower than MPI [25] and therefore more cost-effective. Nevertheless, precise cost-benefit analyses are currently not available, and further studies evaluating the relationship between CTA and MPI in selecting patients for ICA are warranted.

\section{Clinical implications}

The use of CTA to exclude significant CAD may allow cardiologists to restrict referral for ICA to patients in whom the need for interventional therapy is highly likely [26]. In patients with a normal CTA examination CAD can be safely ruled out and the patient may be reassured. Conversely, patients with significant stenosis on CTA should be referred for further evaluation. Furthermore, patients with recurrent or worsening symptoms as well as patients with left main or three-vessel disease on CTA could be directly referred for ICA. In patients with non-significant stenosis on CTA, however, medical therapy and lifestyle interventions may be appropriate and these patients may be excluded from ICA. Nevertheless, in patients with uncertain results, functional analysis could be performed to further guide referral for ICA. Notably, while CTA may aid risk stratification for the presence of CAD in patients with a low-to-intermediate risk profile, CTA may be less useful in patients with known CAD, in whom the need for ICA and interventional therapy is likely $[6,27,28]$.

\section{Limitations}

Several limitations of the present study merit further consideration. Firstly, CTA is inherently associated with ionizing radiation [29]. Secondly, CTA and ICA do not provide information regarding the functional significance of a lesion. Combined anatomic and perfusion imaging using either a hybrid imaging approach or volumetric CTA in a single examination would be advantageous and research is ongoing [30]. Third, the effect of other clinical information, such as perfusion imaging, may have also influenced referral for ICA. However, studying the effects other tests as well as cost-benefit analysis were beyond the scope of this study. Last, the present investigation did not evaluate clinical outcome. Future studies are needed to evaluate the effect of CTA on clinical outcome and healthcare costs.

\section{Conclusion}

The present investigation showed that the results of CTA are strong and independent determinants of subsequent ICA as well as revascularization. Consequently, CTA has the potential to serve as a gatekeeper for ICA to identify patients who are most likely to benefit from revascularization and exclude patients who can safely avoid ICA.

Acknowledgments Dr. de Graaf is co-supported by the Dutch Technology Foundation STW (Utrecht, the Netherlands), applied science division of NWO and the Technology Program of the Ministry of Economic Affairs, grant nr. 10084. Dr. van Velzen is supported by the Netherlands Heart Foundation (The Hague, The Netherlands), grant nr 2007B223. Mr. van Werkhoven is financially supported by a research grant from the Netherlands Society of Cardiology (Utrecht, The Netherlands).

Conflict of interest Dr. Jukema has research grants from and was speaker on (CME accredited) meetings sponsored by Astellas (Tokyo, Japan), Astra-Zeneca (London, United Kingdom), Biotronik (Berlin, Germany), Boston Scientific (Natick, United States), Bristol Myers Squibb (New York, United States), Cordis (Roden, Netherlands), Daiichi Sankyo (Tokyo, Japan), Ely Lilly (Indianapolis, United States), Medtronic (Minneapolis, United States), Merck-ScheringPlough (Whitehouse Station, United States), Pfizer (Groton/New London, United States), Orbus Neich (Hong Kong, China), Novartis (Basel, Switserland), Roche (Basel, Switserland), Servier (Neuillysur-Seine, France). Dr. Bax has research grants from Medtronic (Minneapolis, United States), Boston Scientific (Natick, United States), BMS medical imaging (New York, United States), St. Jude Medical (St. Paul, United States), GE Healthcare (Chalfont St Giles, United Kingdom), Biotronik (Berlin, Germany), and Edwards Lifesciences (Irvine, United States). Dr. Schalij has research grants from 
Boston Scientific (Natick, United States), Medtronic (Minneapolis, United States) and Biotronik (Berlin, Germany).

Open Access This article is distributed under the terms of the Creative Commons Attribution License which permits any use, distribution, and reproduction in any medium, provided the original author(s) and the source are credited.

\section{References}

1. Boden WE, O'Rourke RA, Teo KK et al (2007) Optimal medical therapy with or without PCI for stable coronary disease. N Engl J Med 356(15):1503-1516

2. Hachamovitch R, Hayes SW, Friedman JD et al (2004) Stress myocardial perfusion single-photon emission computed tomography is clinically effective and cost effective in risk stratification of patients with a high likelihood of coronary artery disease (CAD) but no known CAD. J Am Coll Cardiol 43(2):200208

3. Min JK, Shaw LJ, Devereux RB et al (2007) Prognostic value of multidetector coronary computed tomographic angiography for prediction of all-cause mortality. J Am Coll Cardiol 50(12): 1161-1170

4. van Velzen JE, de Graaf FR, Kroft LJ et al (2011) Performance and efficacy of 320-row computed tomography coronary angiography in patients presenting with acute chest pain: results from a clinical registry. Int J Cardiovasc Imaging 21(11):2285-2296

5. van Werkhoven JM, Schuijf JD, Gaemperli $O$ et al (2009) Incremental prognostic value of multi-slice computed tomography coronary angiography over coronary artery calcium scoring in patients with suspected coronary artery disease. Eur Heart J 30(21):2622-2629

6. Taylor AJ, Cerqueira M, Hodgson JM et al (2010) ACCF/SCCT/ ACR/AHA/ASE/ASNC/NASCI/SCAI/SCMR 2010 appropriate use criteria for cardiac computed tomography: a report of the American College of Cardiology Foundation Appropriate Use Criteria Task Force, the Society of Cardiovascular Computed Tomography, the American College of Radiology, the American Heart Association, the American Society of Echocardiography, the American Society of Nuclear Cardiology, the North American Society for Cardiovascular Imaging, the Society for Cardiovascular Angiography and Interventions, and the Society for Cardiovascular Magnetic Resonance. J Am Coll Cardiol 56(22):1864-1894

7. de Graaf FR, Schuijf JD, van Velzen JE et al (2010) Evaluation of contraindications and efficacy of oral Beta blockade before computed tomographic coronary angiography. Am J Cardiol 105(6):767-772

8. de Graaf FR, van Werkhoven JM, van Velzen JE et al (2010) Incremental prognostic value of left ventricular function analysis over non-invasive coronary angiography with multidetector computed tomography. J Nucl Cardiol 17(6):1034-1040

9. Schuijf JD, Pundziute G, Jukema JW et al (2006) Diagnostic accuracy of 64-slice multislice computed tomography in the noninvasive evaluation of significant coronary artery disease. Am J Cardiol 98(2):145-148

10. Schuijf JD, Wijns W, Jukema JW et al (2006) Relationship between noninvasive coronary angiography with multi-slice computed tomography and myocardial perfusion imaging. J Am Coll Cardiol 48(12):2508-2514

11. de Graaf FR, Schuijf JD, van Velzen JE et al (2010) Diagnostic accuracy of 320-row multidetector computed tomography coronary angiography to noninvasively assess in-stent restenosis. Invest Radiol 45(6):331-340
12. Raff GL, Abidov A, Achenbach S et al (2009) SCCT guidelines for the interpretation and reporting of coronary computed tomographic angiography. J Cardiovasc Comput Tomogr 3(2): $122-136$

13. de Graaf FR, Schuijf JD, van Velzen JE et al (2010) Diagnostic accuracy of 320-row multidetector computed tomography coronary angiography in the non-invasive evaluation of significant coronary artery disease. Eur Heart J 31(15):1908-1915

14. Meijboom WB, Meijs MF, Schuijf JD et al (2008) Diagnostic accuracy of 64-slice computed tomography coronary angiography: a prospective, multicenter, multivendor study. J Am Coll Cardiol 52(25):2135-2144

15. Henneman MM, Schuijf JD, van Werkhoven JM et al (2008) Multi-slice computed tomography coronary angiography for ruling out suspected coronary artery disease: what is the prevalence of a normal study in a general clinical population? Eur Heart J 29(16):2006-2013

16. Chow BJ, Abraham A, Wells GA et al (2009) Diagnostic accuracy and impact of computed tomographic coronary angiography on utilization of invasive coronary angiography. Circ Cardiovasc Imaging 2(1):16-23

17. Hulten EA, Carbonaro S, Petrillo SP et al (2011) Prognostic value of cardiac computed tomography angiography: a systematic review and meta-analysis. J Am Coll Cardiol 57(10):1237-1247

18. Bateman TM, O'Keefe JH Jr, Dong VM et al (1995) Coronary angiographic rates after stress single-photon emission computed tomographic scintigraphy. J Nucl Cardiol 2(3):217-223

19. Shaw LJ, Hachamovitch R, Berman DS et al (1999) The economic consequences of available diagnostic and prognostic strategies for the evaluation of stable angina patients: an observational assessment of the value of precatheterization ischemia. Economics of noninvasive diagnosis (END) multicenter study group. J Am Coll Cardiol 33(3):661-669

20. Wijns W, De BB, Vanhoenacker PK (2007) What does the clinical cardiologist need from noninvasive cardiac imaging: is it time to adjust practices to meet evolving demands? J Nucl Cardiol 14(3):366-370

21. Nucifora G, Schuijf JD, van Werkhoven JM et al. (2010) Relationship between obstructive coronary artery disease and abnormal stress testing in patients with paroxysmal or persistent atrial fibrillation. Int J Cardiovasc Imaging 27(6):777-785

22. van Werkhoven JM, Schuijf JD, Gaemperli O et al (2009) Prognostic value of multislice computed tomography and gated single-photon emission computed tomography in patients with suspected coronary artery disease. J Am Coll Cardiol 53(7): 623-632

23. Priest VL, Scuffham PA, Hachamovitch R et al (2011) Costeffectiveness of coronary computed tomography and cardiac stress imaging in the emergency department: a decision analytic model comparing diagnostic strategies for chest pain in patients at low risk of acute coronary syndromes. JACC Cardiovasc Imaging 4(5):549-556

24. Hoilund-Carlsen PF, Johansen A, Christensen HW et al (2006) Potential impact of myocardial perfusion scintigraphy as gatekeeper for invasive examination and treatment in patients with stable angina pectoris: observational study without post-test referral bias. Eur Heart J 27(1):29-34

25. Budoff MJ, Karwasky R, Ahmadi N et al (2009) Cost-effectiveness of multidetector computed tomography compared with myocardial perfusion imaging as gatekeeper to invasive coronary angiography in asymptomatic firefighters with positive treadmill tests. J Cardiovasc Comput Tomogr 3(5):323-330

26. Achenbach S, Daniel WG (2010) Cardiac imaging in the patient with chest pain: coronary CT angiography. Heart 96(15):1241-1246

27. Bluemke DA, Achenbach S, Budoff M et al (2008) Noninvasive coronary artery imaging: magnetic resonance angiography and 
multidetector computed tomography angiography: a scientific statement from the american heart association committee on cardiovascular imaging and intervention of the council on cardiovascular radiology and intervention, and the councils on clinical cardiology and cardiovascular disease in the young. Circulation 118(5):586-606

28. de Graaf FR, Schuijf JD, Scholte AJ et al (2010) Usefulness of hypertriglyceridemic waist phenotype in type 2 diabetes mellitus to predict the presence of coronary artery disease as assessed by computed tomographic coronary angiography. Am J Cardiol 106(12):1747-1753

29. van der Wall EE, Jukema JW, Schuijf JD et al (2011) $100 \mathrm{kV}$ versus $120 \mathrm{kV}$ : effective reduction in radiation dose? Int J Cardiovasc Imaging 27(4):587-591

30. Ko BS, Cameron JD, Meredith IT et al. (2011) Computed tomography stress myocardial perfusion imaging in patients considered for revascularization: a comparison with fractional flow reserve. Eur Heart J 33(1):67-77 